\title{
Predictors of outcome in a large retrospective cohort of patients with transverse myelitis
}

Matteo Gastaldi, Enrico Marchioni, Valeria Mariani, Laura Di Lodovico, Paola Banfi, Roberto Bergamaschi, Enrico Alfonsi, Paola Borrelli, Ottavia Ferraro, Elisabetta Zardini, Anna Pichiecchio Andrea Cortese, Mauro Ceroni, Giorgio Bono, Diego Franciotta

Corresponding Author: Matteo Gastaldi, MD

IRCCS, C. Mondino National Neurological Institute, via Mondino 2, 27100 Pavia, Italy. Tel: 003903823801. Email: matteo.gastaldi@mondino.it

Word count: 3334

Tables: 4 


\section{ABSTRACT}

Background Transverse myelitis (TM) is an inflammatory disorder of the spinal cord that can be idiopathic, or associated with demyelinating diseases, collagen vascular diseases, or post-infectious syndromes. Uncertainty exists on the prognosis of initial TM presentations. We aimed to identify outcome predictors in this pathology.

Methods This is a retrospective study on patients with isolated TM as the first manifestation of neurological disorders. Scores $\geq 3$ on the modified Rankin scale (mRS) marked high disability.

Results 159 patients (mean age, 47 years; $43 \%$ female) were identified and, at the end of follow-up (mean time, 62.2 months), TM was classified as follows: idiopathic (I-TM, n=53), post-infectious (PI-TM, $\mathrm{n}=48$ ), associated with multiple sclerosis (MS-TM, $\mathrm{n}=51$ ), or to neuromyelitis optica spectrum disorder/collagen vascular disease/neurosarcoidosis $(n=7)$. At follow-up, 42 patients were severely disabled, and patients with I-TM or PI-TM showed the worst outcomes (Mean mRS score 2.2 (PI-TM) v 1.6 (I-TM) v 0.7 (MS-TM), p<0.001). Predictors of disability at onset were: infectious antecedents, sphincteric and pyramidal symptoms, high initial mRS scores, presence of blood-cerebrospinal fluid-barrier damage, and lumbosacral MRI lesions (on univariate analysis), and older age (odds ratio (OR), 1.1; 95\%CI, 1.0-1.1), overt/subclinical involvement of the peripheral nervous system (PNS) (OR, 9.4; 95\%CI, 2.2-41.0), complete TM (OR, 10.8; 95\%CI, 3.4-34.5) on multivariate analysis. Conclusions Our findings help define prognosis and therapeutic choices in the first manifestations of TM. Notably, infectious antecedents and the PNS involvement associate with a severe prognosis. TM thorough prognostic assessment should include neurophysiology, nerve conduction studies and lumbosacral MRI.

\section{INTRODUCTION}


Transverse myelitis (TM) is a focal inflammatory disorder of the spinal cord that can occur in idiopathic forms, or within a context of heterogeneous pathologies, mainly demyelinating diseases, collagen vascular diseases, and post-infectious syndromes.[1] Although it is a relatively uncommon disorder, studies aimed to improve diagnosis, assess prognosis, and allow prompt treatments are essential to reduce the disability that affects a considerable portion of TM patients.

Consensus-based efforts have been made to uniform the diagnostic criteria for TM, reduce diagnostic confusion, and lay the basis for multicenter clinical trials.[2] However, the classification schemes resulting from the criteria have nosographic limitations (reviewed in [3]), and accurate etiologic and prognostic assessments of the disorder at onset are still pending, with the exception of the best characterised TM types. MRI and other paraclinical tools are indeed available to support the diagnosis of multiple sclerosis (MS)[4], neuromyelitis optica spectrum disorders (NMOSD)[5], and collagen vascular diseases.[6]

On the other hand, there are very few clinical, biological, and MRI features that, at TM onset, can be strong predictors of long-term disability, such as anti-aquaporin-4 (AQP-4) antibodies, acute onset, complete TM phenotypes, and electromyographic findings of anterior horn involvement.[7, 8] In our previous study on post-infectious neurologic syndromes (PINS), we found that the peripheral nerve system (PNS) comorbidity negatively affects their outcome.[9]

The main aims of this study were the characterization of a large cohort of patients who presented with isolated TM as the first manifestation of disease, and the assessment of predictors of long-term disability.

\section{PATIENTS AND METHODS}


We reviewed the records of adult patients admitted for an initial episode of TM to the Mondino National Neurological Institute (Pavia, Italy), or to the Circolo/Fondazione Macchi Hospital (Varese, Italy) from January 2000 to January 2012. The inclusion criteria were: 1) motor and/or sensory and/or autonomic dysfunctions attributable to spinal cord lesions; 2) clues of inflammation within the spinal cord, as indicated by at least one of the following: presence of gadolinium enhancement on spine MRI, cerebrospinal fluid (CSF) pleocytosis (>5 leukocytes $/ \mathrm{mm}^{3}$ ), elevated IgG index or unique-to-CSF oligoclonal IgG bands (OCB); 3) no anamnestic history of CNS inflammatory disease or optic neuritis; 4) no signs/symptoms of encephalic or brainstem involvement at onset; 5) no radiological evidence of spinal cord compression; 6) no underlying infectious causes; 7) maximum disability reached in $>4$ hours, with symptoms lasting >48 hours. The exclusion criteria were: 1 ) evidence of neoplastic or ischemic etiology; 2) follow-up of less than 2 years in patients with MS. At the end of follow-up, patients were classified in 4 different groups according to the final diagnosis: 1) isolated TM as the first manifestation of MS (MS-TM) [10]; 2) TM associated with other autoimmune diseases such as collagen vascular diseases or anti-AQP-4-seropositive NMOSD;[11-13] 3) post-infectious TM (PI-TM), when an infectious prodromal event had occurred within three weeks from the TM onset and the diagnostic criteria for the first two groups were not fulfilled (this group included post-vaccine TM); 4) idiopathic TM (I-TM), when none of the diagnostic criteria for the above-mentioned disorders were fulfilled.

The definition of prodromal infectious event required the presence of fever (body temperature $\geq 38^{\circ} \mathrm{C}$ ), isolated or associated with flu-like, urinary tract, respiratory and/or gastrointestinal symptoms. The type of TM (partial versus complete) was defined according to previously published criteria.[3] Disability was measured with the modified Rankin scale (mRS) at disease onset and at the end of follow-up; high disability was defined by an $\mathrm{mRS}$ score $>2$.[14] A relapse was defined as an increase of disability of at least 1 point of $\mathrm{mRS}$ 
score, associated withwith CSF and/or MRI evidence of inflammation. Laboratory data included CSF cell number and type, an indicator of blood-CSF-barrier (B-CSF-B) damage that measures the degree of CNS inflammation, [15] IgG Index and OCB to assess the intrathecal IgG synthesis,[15] and anti-AQP-4 antibodies. OCB were detected in serum and CSF with isoelectric focusing, and classified in accordance to standardized patterns.[15] An expert neuroradiologist (A.P.) reviewed the spinal cord and brain MRI scans, which were examined for the presence of inflammatory lesions (scored according to the Barkhof criteria.[4]) Dissemination in space and time was assessed according to the McDonald criteria.[10] Spinal lesions spanning >2 vertebral segments identified longitudinally extensive TM (LETM).[5] Electrophysiological data on visual evoked potentials (VEP) and nerve conduction studies (NCS) were collected when available. Following the recommendations of our previous study on PINS,[9] the group of patients with PI-TM underwent NCS systematically as part of a routine diagnostic protocol. The PNS involvement was defined as the presence of: a) spinal root contrast enhancement on spine MRI, and/or b) NCS abnormalities with demyelinating features (otherwise, in the presence of isolated axonal features, the Hadden criteria for acute motor axonal neuropathy had to be fulfilled.[16]) Patients with peripheral nerve diseases that preexisted the TM onset, or with known risk factors for these diseases (e.g. diabetes, $\mathrm{HCV}$ infection, monoclonal gammopathy) were not included in the group of those with concomitant TM and PNS involvement. Follow-up information were collected from outpatient clinic reports or, when unavailable, with telephonic interviews.

The Institutional Review Boards approved the conduct of this study (standard protocol approvals, registrations, and patient consents).

\section{Anti-MOG antibody testing}


Anti-myelin oligodendrocyte glycoprotein (MOG) antibodies have been recently associated withwith NMOSD-like phenotypes.[17] Sera of 46 patients (PI-TM, 24; I-TM, 12; anti-AQP4-seropositive NMOSD, 2; sarcoidosis, 1; MS, 7), which were collected near to the first TM episode and stored at $-80^{\circ} \mathrm{C}$, were available for anti-MOG antibody determination. Tests were performed at the Autoimmune Neurology Laboratory, Oxford (UK), with a live cell-based assay expressing the full-length human MOG.[18]

\section{Statistical analysis}

Mean values and standard deviations (SD) were used for the quantitative variables, and percentages for the categorical ones. Comparisons among the four groups and categorical variables were evaluated with the chi-square test, Fisher's exact test, or Fisher-FreemanHalton test. The parametric test for analysis of variance (ANOVA) or the corresponding nonparametric Kruskal-Wallis rank test were used for the quantitative variables. Contrasts between couple of groups was performed and the Bonferroni correction for multiple comparisons was applied. Normality distribution was assessed with the Shapiro-Wilk test. To evaluate the associations between endpoint variables (mRS scores at the end of follow-up) and clinico-radiological variables at onset of the disease, odds ratios (ORs) and the corresponding $95 \% \mathrm{CI}$ were calculated. A logistic regression analysis was carried out to identify the mutually adjusted effect among endpoints and the independent variables chosen on the basis of the statistics significance (univariate analysis, $\mathrm{p}<0.05$ ) and of the clinical judgement. P-value $\leq 0.05$ was considered significant (two-sided). All the analyses were conducted with STATA/SE for Windows, version 14.2 (StataCorp, Stata Statistical Software: Release 14. College Station, TX: StataCorp LP).

\section{RESULTS}


We identified 170 patients fulfilling the inclusion criteria; 11 patients were subsequently excluded due to alternative diagnoses (spinal cord tumor, $n=2$; spinal cord infarction, $n=4$ ), or a lack of complete data $(n=5)$. Therefore, 159 patients were included in the study and classified in groups, in accordance with the final diagnosis, as follows: PI-TM ( $\mathrm{n}=48)$, I-TM $(\mathrm{n}=53)$; MS-TM ( $\mathrm{n}=51)$; miscellaneous ( $\mathrm{n}=7$, namely anti-AQP-4-seropositive NMOSD, $\mathrm{n}=2$; sarcoidosis, $\mathrm{n}=2$; systemic sclerosis associated with anti-AQP-4 antibodies, $\mathrm{n}=1$; undifferentiated connective tissue disorder, $\mathrm{n}=1$; systemic lupus erythematosus associated with anti-AQP-4 antibodies, $n=1$ ). Table 1 shows their clinico-demographic characteristics. Age at presentation was significantly lower in the MS group. The mean follow up was 62.2 months (range, 4-220).

Table 1: Clinico-demographic characteristics of the cohort of patients with transverse myelitis

\begin{tabular}{lcccc}
\hline & $\begin{array}{c}\text { All patients, } \mathrm{n}(\%) \\
159(100)\end{array}$ & $\begin{array}{c}\text { PI-TM, }(\%) \\
48(30.2)\end{array}$ & $\begin{array}{c}\text { I-TM, n }(\%) \\
53(33.3)\end{array}$ & $\begin{array}{c}\text { Miscellaneous, } \mathrm{n} \\
(\%)\end{array}$ \\
& & & & $7(4.4)$ \\
\hline Age at presentation, mean (SD) & $46.7(17.2)$ & $53.8(18.3)$ & $51.26(16.2)$ & $48.57(13.6)$ \\
Months of follow up, mean (SD) & $62.2(37.8)$ & $58.5(31.4)$ & $69.1(40.1)$ & $109.1(63.5)$ \\
Males & $69(43.4)$ & $27(56.2)$ & $19(35.9)$ & $1(14.3)$ \\
PNS involvement & $20(12.6)$ & $13(27.1)$ & $7(13.2)$ & $0(0)$ \\
Prodromal infectious events & $51(32.1)$ & $48(100)$ & $0(0)$ & $1(14.3)$ \\
TM type & & & & \\
$\quad$ Complete & $30(18.9)$ & $20(41.7)$ & $9(17)$ & $1(14.3)$ \\
$\quad$ Partial & $129(81.1)$ & $28(58.3)$ & $44(83)$ & $6(85.7)$ \\
Signs/symptoms & & & & \\
$\quad$ Sphincteric & $75(47.2)$ & $39(81.2)$ & $22(41.5)$ & $4(57.1)$ \\
Pyramidal & $100(62.9)$ & $34(70.8)$ & $38(71.7)$ & $5(71.4)$ \\
$\quad$ Sensory & $156(98.1)$ & $47(97.9)$ & $51(96.2)$ & $7(100)$ \\
mRS, presentation, mean (SD) & $2.4(1.4)$ & $3.4(1.3)$ & $2.3(1.3)$ & $2.9(0.9)$ \\
mRS, follow-up end, mean (SD) & $1.5(1.6)$ & $2.2(1.7)$ & $1.6(1.7)$ & $1.4(1.0)$ \\
\#Relapsing course & $23 / 108(21.3)$ & $6(12.5)$ & $11(20.8)$ & $6(85.7)$ \\
\#AP-4 Ab-positive & $4 / 76(5.3)$ & $0 / 24(0)$ & $0 / 27(0)$ & $4 / 7(57.1)$ \\
Treatments & & & \\
$\quad$ Steroids & $147(92.5)$ & $43(89.6)$ & $49(92.5)$ & $7(100)$ \\
IvIg & $22(13.8)$ & $12(25.0)$ & $8(15.1)$ & $2(28.6)$ \\
Azathioprine & $9 y$ clophosphamide & $4(8.3)$ & $2(3.8)$ & $3(42.9)$ \\
Death & $2(1.3)$ & $0(0)$ & $2(3.8)$ & $0(0)$ \\
& $6(3.8)$ & $4(8.3)$ & $2(3.8)$ & $0(0)$ \\
$\quad$ & & &
\end{tabular}

TM, transverse myelitis; n, number; PI-TM, post-infectious TM; I-TM, idiopathic TM; MS-TM, TM in multiple sclerosis; SD, standard deviation; PNS, peripheral nervous system; mRS, modified Rankin 
Scale; NA, not applicable; AQP-4 Ab, anti-aquaporin-4 antibodies; IvIg, intravenous immunoglobulins; \#, number on available patients/samples

Forty-one patients had had infectious or vaccinal antecedents: isolated fever $(n=14)$; flu-like syndrome $(n=12)$; organ-related symptoms with or without fever $(n=15$; respiratory, $n=5$; gastroenteric, $n=7$; urinary, $n=3$ ). In 3 cases, the serological confirmation of infection was available (varicella zoster virus, measles virus, influenza virus). Eight patients had been vaccinated within 4 weeks before the onset of TM (influenza virus, $n=6$; mumps-measlesrubella, $\mathrm{n}=1$; streptococcus pneumoniae, $\mathrm{n}=1$ ).

Almost all the patients presented with sensory symptoms, whereas the frequencies of the sphincteric involvement (81\%), and of the presence of complete TM (42\%) in the PI-TM group were significantly higher than in the other groups. Globally, complete TM affected $19 \%$ of the non-MS patients (Table 1).

Lumbar puncture was performed in 154 patients. The mean value of CSF cells in the PI-TM group (34.5 leukocytes $/ \mathrm{mm}^{3}$; range, 0-144) was significantly higher than in the others. A moderate to severe B-CSF-B damage, which measures the degree of the CNS inflammation, was consistently found in one third of patients with PI-TM and in only two patients with I-TM. As expected, the MS-TM group showed the highest frequency of uniqueto-CSF OCB detection. There were no between-group differences in the other isoelectric focusing patterns (in particular, the mirror or the mixed pattern, which are conventionally associated with systemic or recent intrathecal humoral immune activation, respectively)[15](Table2).

Table 2: Cerebrospinal fluid and neuroradiological findings 


\begin{tabular}{|c|c|c|c|c|}
\hline \multirow[t]{13}{*}{ CSF analysis } & Lumbar puncture performed & $154(96.9)$ & $48(100)$ & $52(98.1)$ \\
\hline & Leukocytes $/ \mathrm{mm}^{3}$, mean $(\mathrm{SD})$ & $15.3(26.3)$ & $34.5(38.6)$ & $7.3(12.4)$ \\
\hline & ${ }^{\# B l o o d-C S F-b a r r i e r ~ d a m a g e ~}$ & & & \\
\hline & None & $74 / 144(51.4)$ & 9/47 (19.1) & $27 / 48(56.2)$ \\
\hline & Mild & $53 / 144(36.8)$ & 23/47 (48.9) & $18 / 48(37.5)$ \\
\hline & Moderate & $12 / 144(8.3)$ & $11 / 47(23.4)$ & $1 / 48(2.1)$ \\
\hline & Severe & $5 / 144(3.5)$ & 4/47 (8.5) & $1 / 48(2.1)$ \\
\hline & IgG Index $>0.70$ & $39 / 148(26.4)$ & $5 / 47(10.6)$ & $10 / 47(21.3)$ \\
\hline & ${ }^{\#}$ IEF patterns & & & \\
\hline & Polyclonal & $53 / 153(34.6)$ & 23/48 (47.9) & $21 / 52(40.4)$ \\
\hline & Mirror & $11 / 153(7.2)$ & $5 / 48(10.4)$ & $4 / 52(7.7)$ \\
\hline & Unique-to- $C S F O C B$ & $75 / 153(49.0)$ & $12 / 48(25.0)$ & $24 / 52(46.2)$ \\
\hline & Mixed & $14 / 153(9.2)$ & $8 / 48(16.7)$ & $4 / 52(7.7)$ \\
\hline \multirow[t]{10}{*}{ Spinal cord MRI } & Multifocal lesions & $54(34.0)$ & $16(33.3)$ & $18(34.0)$ \\
\hline & Lesion sagittal localization & & & \\
\hline & Cervical & $93(58.5)$ & $19(39.6)$ & $28(52.8)$ \\
\hline & Thoracic & $84(52.8)$ & $27(56.2)$ & $33(62.3)$ \\
\hline & Lumbar & $16(10.1)$ & $10(20.8)$ & $4(7.6)$ \\
\hline & LETM & $26(16.4)$ & $8(16.7)$ & $11(20.8)$ \\
\hline & ${ }^{\#}$ Lesion axial localizations & & & \\
\hline & Postero-lateral & $49 / 123(39.8)$ & $6 / 39(15.4)$ & $11 / 38(28.9)$ \\
\hline & Centro-medullar & 16/123 (13) & $6 / 39(15.4)$ & $6 / 38(15.8)$ \\
\hline & ${ }^{\#}$ Contrast enhancement & $95 / 152(62.5)$ & $24 / 48(50)$ & $31 / 51(60.8)$ \\
\hline \multirow[t]{5}{*}{ Brain MRI } & Brain lesions & $64(40.3)$ & $10(20.8)$ & $10(18.9)$ \\
\hline & Barkhof criteria & & & \\
\hline & None & $103(64.8)$ & $40(83.3)$ & $46(86.8)$ \\
\hline & 1 & $12(7.5)$ & $3(6.2)$ & $0(0)$ \\
\hline & $>1$ & $44(27.7)$ & $5(10.4)$ & $7(13.2)$ \\
\hline
\end{tabular}

TM, transverse myelitis; n, number; PI-TM, post-infectious TM; I-TM, idiopathic TM; MS-TM, TM in multiple sclerosis; CSF, cerebrospinal fluid; IEF, isoelectric focusing; OCB, oligoclonal bands; MRI, magnetic resonance imaging; LETM, longitudinally extensive transverse myelitis; \#, number on available reports

However, 36 TM patients that did not develop MS had CSF OCB in the acute phase of the disease, thus confirming the low analytical specificity of this abnormality[15]. During the follow-up of the 40 patients who repeated a lumbar puncture, only 5 showed variations of the initial patterns: unique-to-CSF OCB disappeared in 3 patients with PI-TM; the conversion to unique-to-CSF OCB from a polyclonal and from a mixed pattern respectively occurred in 2 patients with MS.

On spinal cord MRI, the frequency of sagittal cervical localization of the lesions in the MS group (80\%) was significantly higher than in the other groups. Lumbosacral lesions 
were uncommon in all the groups, but, again, mostly detected in patients with PI-TM. Almost all the patients underwent the brain MRI, which showed white matter lesions in the large majority of patients that converted to definite MS; in $63 \%$ of these patients the lesion distribution fulfilled more than one Barkhof criteria (Table 2).

Anti-AQP-4 antibodies, which were tested in nearly half of the patients, were positive in the four patients with NMOSD. All the serum samples tested for anti-MOG antibodies were negative.

Most patients received high-dose intravenous steroids as first-line therapy. Ranked in order of frequency of use, second-line therapies were: intravenous immunoglobulins, azathioprine, and cyclophosphamide (Table 1).

At the end of follow-up, 85 patients showed a monophasic disease, 53 converted to definite MS within a mean time of 11 months (range 1-132), and 23 showed a relapsing course. Given the typical relapsing course of MS, patients with MS-TM were excluded from the evaluation of predictors of relapse (Table 1). Factors associated with relapses were antiAQP-4 antibody positivity ( $\mathrm{p}=0.001), \mathrm{CSF}$ leukocyte counts $<10$ cells $/ \mathrm{mm}^{3}(\mathrm{p}<0.001)$, having a diagnosis of LETM ( $\mathrm{p}=0.006)$, or of PI-TM $(\mathrm{p}<0.001)$.

The assessment of the PNS was a mainstay of our study. NCS were performed in 72 patients (45\%), more frequently in the PI- (70\%) and I-TM groups (43\%) than in the MS group (32\%). Twenty patients, 13 with PI-TM and 7 with I-TM, had the PNS involvement (Table 3).

Table 3: Characteristics of patients with transverse myelitis and the peripheral nervous system involvement

\begin{tabular}{llc}
\hline & & Patients, $\mathrm{n}(\%)$ \\
& & $(20)$ \\
\hline Clinical findings & Age, mean (range) & $60(47-79)$ \\
& Males & $9(45)$
\end{tabular}


Spinal roots enhancement $2(10)$

Abnormal NCS 8 (40)

Spinal root enhancement plus abnormal NCS 10 (50)

Groups

PI-TM $13(65)$

I-TM 7 (35)

Miscellanea $\quad 0(0)$

$M S-T M \quad 0(0)$

Symptoms/signs suggestive of PNS involvement
Hypo/areflexia

Sock and glove paresthesias $5(25)$

Perineal anesthesia 3 (15)

None $4(20)$

Relapsing course $3(15)$

Death $3(15)$

Neurophysiology NCS features

Demyelinating 7 (35)

Axonal 7 (35)

Mixed $4(20)$

Normal 2 (10)

Hadden criteria

AIDP $1(5)$

AMAN $3(15)$

$\begin{array}{ccc}\text { CSF analysis } & \begin{array}{c}\text { Blood-CSF-barrier damage } \\ \text { Mild }\end{array} & 8(40)\end{array}$

Moderate $5(25)$

Severe $2(10)$

None $\quad 5(25)$

Albuminocytologic dissociation 2 (10)

IEF patterns

Polyclonal $8(40)$

Mirror $2(10)$

Unique-to-CSF OCB 6 (30)

Mixed 4 (20)

$\begin{array}{ll}\text { Spinal cord MRI } & \begin{array}{l}\text { Lesion localizations } \\ \text { Cervical }\end{array} \\ \text { Thoracic (20) }\end{array}$

Thoracic $8(40)$

Lumbosacral $8(40)$

n, number; PNS, peripheral nervous system; NCS, nerve conduction study; PI-TM, post-infectious transverse myelitis; I-TM, idiopathic TM; MS-TM, TM in multiple sclerosis; AIDP, acute inflammatory demyelinating polyradiculoneuropathy; AMAN, acute motor axonal neuropathy; CSF, cerebrospinal fluid; IEF, isoelectric focusing; OCB, oligoclonal bands; MRI, magnetic resonance imaging

Twenty patients showed the PNS involvement, which relied only on NCS abnormalities in 8 of them. In this subgroup, the neuropathy was demyelinating in 4 cases ( 1 fulfilling the criteria for acute inflammatory demyelinating polyneuropathy), axonal in 2 (both fulfilling the criteria for acute motor axonal neuropathy),[16] and mixed axonal and demyelinating in 
2. Signs or symptoms, alone or in combination, which suggested the PNS involvement included: reduced or absent deep tendon reflexes (11 patients), sock and glove paresthesias (5 patients), and perineal anesthesia (3 patients). Interestingly, 4 patients did not present any symptom of peripheral neuropathy. Almost all the patients with the PNS involvement showed CSF inflammatory signs, as demonstrated by moderate or severe B-CSF-B damage, and bad outcomes at the end of follow up (including 3 deaths from infections).

As for the disability, at onset the mean mRS scores of patients with PI-TM (3.4) and I-TM (2.2) were significantly higher than those of the MS patients (1.5). During the acute phase of the disease $26.4 \%$ of the TM patients showed high disability: 37 patients were bedridden (22 of whom had PI-TM), and 22 needed assistance to walk. At the end of followup, 42 patients still had mRS scores $>2$, mostly belonging to the PI- (mean value, 2.2, range?) and I-TM groups (mean value, 1.6, range). No patients with TM-MS presented mRS scores $>3$ at the end of follow-up (mean value, 0.7 , range; $p<0.001$ ).

We then analysed a list of clinico-laboratory variables possibly associated with bad outcomes in all the studied groups at the end of follow-up (Table 4).

Table 4: Outcome predictors in the cohort of patients with transverse myelitis

\begin{tabular}{|c|c|c|c|c|c|c|}
\hline & \multicolumn{3}{|l|}{ Outcome } & \multicolumn{3}{|c|}{ Univariate analysis } \\
\hline & $\begin{array}{c}\text { Good, n (\%) } \\
117(73.6)\end{array}$ & $\begin{array}{c}\text { Bad, n (\%) } \\
42(26.4)\end{array}$ & p-value & OR & CI $(95 \%)$ & p-value \\
\hline Mean age at onset, years (SD) & $41.6(15.4)$ & $60.8(13.7)$ & $<0.001$ & 1.07 & $1.05-1.09$ & $<0.001$ \\
\hline Males & $51(43.6)$ & $18(42.9)$ & 0.90 & 0.97 & $0.5-1.98$ & 0.93 \\
\hline Prodromal infectious events & $29(24.8)$ & $22(52.4)$ & $<0.001$ & 3.34 & $1.55-7.18$ & 0.001 \\
\hline Complete TM & $8(6.8)$ & $22(52.4)$ & $<0.001$ & 14.99 & $5-44.92$ & $<0.001$ \\
\hline \multicolumn{7}{|l|}{ Signs/symptoms } \\
\hline Sphinteric & $42(35.9)$ & $33(78.6)$ & $<0.001$ & 6.6 & $2.7-16.1$ & $<0.001$ \\
\hline Pyramidal & $61(52.1)$ & $39(92.9)$ & $<0.001$ & 11.9 & $3.2-45.0$ & $<0.001$ \\
\hline Sensory & $115(98.3)$ & $41(97.6)$ & 0.90 & 0.7 & $0.1-8.1$ & 0.78 \\
\hline mRS at onset, mean (SD) & $1.84(0.93)$ & $3.93(1.07)$ & $<0.001$ & 3.2 & $2.5-4.2$ & $<0.001$ \\
\hline Relapsing TM & $13(11)$ & $10(23.8)$ & 0.04 & 2.5 & $1.0-6.3$ & 0.044 \\
\hline PNS involvement & $3(2.6)$ & $17(40.5)$ & $<0.001$ & 25.8 & $5.7-117.2$ & $<0.001$ \\
\hline CSF cells, $>10$ leukocytes $/ \mathrm{mm}^{3}$ & $32(29.1)$ & $17(40.5)$ & 0.13 & 1.6 & $0.78-3.5$ & 0.18 \\
\hline Blood-CSF-barrier damage & $5(4.9)$ & $12(29.3)$ & $<0.001$ & 8.11 & $2.5-26.9$ & $<0.001$ \\
\hline
\end{tabular}


Moderate/severe

Lesion localizations on MRI

Cervical

Thoracic

$74(63.2)$

$19(45.2)$

0.04

0.5

$0.2-1.0$

0.043

Lumbosacral

$61(52.1)$

$23(54.8)$

0.77

1.1

$0.6-2.3$

0.771

LETM

$6(5.1)$

$10(23.8)$

$<0.001$

5.8

$1.9-17.9$

$<0.001$

$15(12.8)$

$11(26.2)$

2.4

$1.0-5.9$

0.045

n, number; OR: odds ratio; CI, confidence interval; TM, transverse myelitis; mRS, modified Rankin scale; PNS, peripheral nervous system; CSF, cerebrospinal fluid; MRI, magnetic resonance imaging; LETM, longitudinally extensive transverse myelitis

In univariate analysis, clinical risk factors for high disability were: older age at onset, infectious antecedents (i.e., to belong to the PI-TM group), complete TM, the PNS involvement, relapsing courses, sphincteric and pyramidal symptoms. In particular, the PNS involvement emerged as a strong disability predictor, with an OR of 25.8 (95\% CI: 5.7117.2). As for the CSF analysis, the presence of moderate-to-severe B-CSF-B damage was the only disability predictor. Finally, spine MRI-detected lumbosacral lesions and the presence of LETM were associated with bad outcomes.

In the multivariate model, the following factors were identified as independent predictors of disability: a) age at presentation [OR, 1.1 (95\%CI 1.0-1.1)], b) the PNS involvement [OR, 9.4 (95\%CI 2.2-41.0)], and c) complete TM [OR, 7.8 (95\%CI 2.5-23.7)] (Table 4).

\section{DISCUSSION}

Based on consistent data from a large cohort of patients with initial, isolated TM and longterm follow-ups, our study confirms that this syndrome often has a severe prognosis, as bad outcomes (an EDSS of $>\ldots$ ) occurred in about one fourth of the cases. We found that high disability at onset, complete TM, motor and urinary symptoms were the strongest predictors of bad outcomes, in accordance with the results of previous studies.[7, 19] In turn, we identified, among others, additional risk factors for high disability, such as the PNS 
involvement, the presence of prodromal infectious events, and of moderate/severe bloodCSF-barrier damage on the CSF analysis.

Interestingly, the PNS involvement emerged as a main negative prognostic factor in both univariate and multivariate analysis. This finding is not completely new, since a) in one series of patients with non-MS-TM, the prevalence of EMG/NCS abnormalities was $27 \%,[20]$ slightly higher than that we found in our series (20\%); however, comparable to the present study, Harzheim and colleagues used less stringent criteria for the PNS involvement definition, and reported no data on long-term outcomes, which could allow reliable evaluations of the clinico-paraclinical variables that they considered to be prognostically important;[20] b) in another series of patients with acute TM, neurophysiology abnormalities associated with bad outcome, but they were substantially related to the severity of the spinal cord anterior horn involvement.[8] In our series, the patients showed enhancement of the spinal roots on MRI, NCS abnormalities, or both. In only 2 of the 8 patients who presented with demyelinating-axonal mixed features, without a better explanation than the TM association, the anterior horn damage could not be excluded. Notably, in $20 \%$ of TM patients with the PNS involvement the clinical picture was dominated by myelopathy signs and symptoms that concealed the clinical manifestations of the PNS damage, which was thus detected following standard diagnostic protocols of our hospital. This observation boldly suggests that EMG/NCS studies should be part of the diagnostic iter in TM, to characterize the extent of the inflammatory process, assess prognosis, assist therapeutic choices, and contribute to the differential diagnosis with MS.

Infectious or vaccinal antecedents associated with the highest frequency of worse outcomes, as near a half of the PI-TM patients showed mRS scores $>2$. As a collateral finding, $75 \%$ of the patients with the PNS involvement had PI-TM, and, as a result, the worst prognosis among our TM cohort. These patients also showed lumbosacral lesions and 
sphincteric symptoms, similarly to what reported in previous studies.[20, 22]. However, the percentages of PI-TM patients among other TM case series are rather variable, ranging from $6 \%$ to $46 \%,[1,21]$ as expected due to the lack of consensus criteria for the nosographic definition of the post-infectious neurological disorders. In our previous study on these syndromes, which can affect CNS, PNS, or both, the PNS involvement was the only independent predictor of relapse.[15] This finding has not been confirmed by the present study, as only $15 \%$ of patients with the PNS involvement showed relapsing disease courses.

Seropositivity for anti-AQP-4 antibodies and LETM associated with relapses, as expected as previously reported.[22] On the contrary, strong predictors of monophasic disease were infectious antecedents and CSF findings of high leukocyte counts.

The presence of complete TM was another unsurprising feature associated with bad outcomes within the groups of patients with PI- and I-TM. Complete TM also predicted the lack of conversion to MS, since no patient with this feature developed MS after sufficiently long follow-up periods. These findings, together with those on the percentage of conversion to MS that we found in patients with partial TM (about 50\%), are cumulatively in line with the literature data.[19, 23] The high frequency of complete TM also accounts for the degree of disability at onset and at the end of follow-up that was higher in the PI- and I-TM groups than in the MS group. On the contrary, Sellner and colleagues found that the EDSS scores in patients who developed MS were higher than those in patients with TM,[24] but this apparent discrepancy is likely due to the exclusion of patients with complete TM from their series.

In full accordance with the respective pathology characteristics, the presence of inflammatory lesions on the brain MRI at onset was the single factor more frequently associated with conversion to MS, as widely reported.[19, 23-25] However, brain lesions fulfilling more than 2 of the Barkhof criteria, which should allow earlier diagnosis of MS,[4] were found in only half of the patients belonging the MS group. 
Analogously to the brain MRI lesions, the presence of CSF OCB strongly associated with conversion to MS, being detected in the MS group with a frequency which was similar to that reported in other studies, $[19,25]$ and notwithstanding that one third of our CSF OCBpositive patients did not have MS. The presence of alternative OCB patterns, namely 'mirror' or 'mixed' patterns, did not help differentiate MS from PI- and I-TM. Interestingly, our findings on patients who underwent a second lumbar puncture during follow-up showed changes of the OCB pattern in some of them, in line with the pathogenetic mechanisms underlying the different TM phenotypes.[26] The repetition of lumbar puncture may thus offer useful information in TM cases with uncertain diagnosis.

CSF analysis provided useful information on the degree of blood-CSF-barrier damage. Moderate/severe damage was absent in patients with MS-TM, and more frequent in those PI- and I-TM patients who showed higher disability.

Anti-AQP-4 and anti-MOG antibodies have been detected in patients with clinical signs and neuroimaging pictures of TM, although how such antibody reactivities to so different antigens can yield overlapping phenotypes is still unclear.[27] In our series, four patients were positive for anti-AQP-4 antibodies (5\%). The low number of patients prevented any statistical analysis. We found no seropositivity for anti-MOG antibodies, likely due to the small number of the available serum samples of patients with LETM, namely one of the adult phenotype that frequently associates to these antibodies.[17]

As for the treatments, our patients with the PNS involvement were poor responders, and required prolonged steroids use. On the contrary, DeSena and colleagues found that patients with concomitant myelitis and polyradiculoneuritis responded well to the treatments, but the case series was small, and, above all, the patients aged under 18.[28]

The retrospective nature of the study entails limitations, such as the impossibility of adequate evaluations of the treatment efficacy due to their heterogeneity in regimens and 
doses, the lack of the full appreciation of the PNS involvement, since the neurophysiology protocol was performed exclusively in the Neurological Institute of Pavia, and the relatively high number of PI-TM patients, who were unbalanced vs I-TM, as the Neurological Institute of Pavia is a referral hospital for the PINS.

In conclusion, our data contribute to a better prognostic definition of isolated and initial TM, in part confirming previous observations, in part adding new information on the negative prognostic role of infectious antecedents and of the PNS involvement, either overt, or asymptomatic. While waiting for confirmation from prospective studies, the improvement in the prognostic assessment can help neurologists in the therapeutic choices.

\section{Acknowledgments}

Supported by the Italian Ministry of Health (RF-2011-02347955), and by RC 2017-2019 funds of the National Neurological Institute C. Mondino. Thanks Alfredo Romani, Paola Bini, Luca Diamanti,

\section{References}

1. De Seze J, Stojkovic T, Breteau G et al. Acute Myelopathies. Clinial, laboratory and outcome profiles in 79 cases. Brain. 2001;124:1509-21.

2. Group TMCW. Proposed diagnostic criteria and nosology of acute transverse myelitis. Neurology. 2002;59:499-505.

3. Scott TF. Nosology of idiopathic transverse myelitis syndromes. Acta Neurol Scand. 2007;115:371-6.

4. Barkhof F, Filippi M, Miller D. Comparison of MRI criteria at first presentation to predict conversion to clinically definite multiple sclerosis. Brain. 1997;120:2059-69.

5. Wingerchuk DM, Banwell B, Bennett JL et al. International consensus diagnostic criteria for neuromyelitis optica spectrum disorders. Neurology. 2015;85:177-89. 
6. Op De Beeck K, Vermeersch P, Verschueren P et al. Antinuclear antibody detection by automated multiplex immunoassay in untreated patients at the time of diagnosis. Autoimmun Rev. 2012;12:137-43.

7. Debette S, de Seze J, Pruvo JP et al. Long-term outcome of acute and subacute myelopathies. J Neurol. 2009;256:980-8.

8. Kalita J, Misra U, Mandal S. Prognostic predictors of acute transverse myelitis. Acta Neurol Scand. 1998;98.

9. Marchioni E, Ravaglia S, Montomoli C et al. Postinfectious neurologic syndromes: a prospective cohort study. Neurology. 2013;80:882-9.

10. Polman CH, Reingold SC, Banwell B et al. Diagnostic criteria for multiple sclerosis: 2010 revisions to the McDonald criteria. Ann Neurol. 2011;69:292-302.

11. Hochberg MC. Updating the American College of Rheumatology revised criteria for the classification of systemic lupus erythematosus. Arthritis Rheum. 1997;40:1725.

12. Petri M, Orbai AM, Alarcon GS et al. Derivation and validation of the Systemic Lupus International Collaborating Clinics classification criteria for systemic lupus erythematosus. Arthritis Rheum. 2012;64:2677-86.

13. Vitali C, Bombardieri S, Jonsson R et al. Classification criteria for Sjogren's syndrome: a revised version of the European criteria proposed by the American-European Consensus Group. Ann Rheum Dis. 2002;61:554-8.

14. Cobo Calvo A, Mané Martinez M, Aletorn-Palau A, Escuer J, Romero Pinel L, MartinezYelamo S. Idiopathic acute transverse myelitis: outcome and conversion to multiple sclerosis in a large series. BMC Neurology. 2013;13.

15. Andersson M, Alvarez-Cermeno J, Bernardi G et al. Cerebrospinal fluid in the diagnosis of multiple sclerosis: a consensus report. J Neurol Neurosurg Psychiatry. 1994;57:897-902. 16. Hadden R, Conrnblath D, Huges R. Electrophysiological classification of Guillain-Barré syndrome: clinical associations and outcome. Ann Neurol. 1998;44:780-88.

17. Kitley J, Woodhall M, Waters P et al. Myelin-oligodendrocyte glycoprotein antibodies in adults with a neuromyelitis optica phenotype. Neurology. 2012;79:1273-7.

18. Waters P, Woodhall M, O'Connor KC et al. MOG cell-based assay detects non-MS patients with inflammatory neurologic disease. Neurol Neuroimmunol Neuroinflamm. 2015;2:e89.

19. Bourre B, Zephir E, Ongangna J et al. Long-term follow up of Acute partial Transverse Myelitis. Arch Neurol. 2012;69:357-62. 
20. Harzheim M, Schlegel U, Urbach H, Klockgether T, Schmidt S. Discriminatory features of acute transverse myelitis: a retrospective analysis of 45 patients. Journal of the Neurological Sciences. 2004;217:217-23.

21. Jeffery D, Mandler R, Davis L. Retrospective Analysis of 33 Cases, With Differentiation of Cases Associated With Multiple Sclerosis and Parainfectious Events. Arch Neurol. 1993;50:532-5.

22. Kimbrough DJ, Mealy MA, Simpson A, Levy M. Predictors of recurrence following an initial episode of transverse myelitis. Neurol Neuroimmunol Neuroinflamm. 2014;1:e4. 23. Cordonnier C, de Seze J, Breteau G et al. Prospective study of patients presenting with acute partial transverse myelopathy. J Neurol. 2003;250:1447-52.

24. Sellner J, Luthi N, Buhler R et al. Acute partial transverse myelitis: risk factors for conversion to multiple sclerosis. Eur J Neurol. 2008;15:398-405.

25. Young J, Quinn S, Hurrell M, Taylor B. Clinically isolated acute transverse myelitis: prognostic features and incidence. Mult Scler. 2009;15:1295-302.

26. Franciotta D, Columba-Cabezas S, Andreoni L et al. Oligoclonal IgG band patterns in inflammatory demyelinating human and mouse diseases. J Neuroimmunol. 2008;200:125-8. 27. Zamvil SS, Slavin AJ. Does MOG Ig-positive AQP4-seronegative opticospinal inflammatory disease justify a diagnosis of NMO spectrum disorder? Neurol Neuroimmunol Neuroinflamm. 2015;2:e62.

28. DeSena A, Graves D, Morriss MC, Greenberg BM. Transverse myelitis plus syndrome and acute disseminated encephalomyelitis plus syndrome: a case series of 5 children. JAMA Neurol. 2014;71:624-9. 